\title{
A Novel Concept for Reliability Evaluation Using Multiple Deterministic Analyses
}

\author{
Reda Farag ${ }^{1,2} \cdot$ Achintya Haldar $^{3}$
}

Received: 29 August 2016/Accepted: 24 October 2016/Published online: 7 November 2016

(C) Indian National Academy of Engineering 2016

\begin{abstract}
A novel reliability analysis concept for large Structural/Mechanical systems represented by finite elements using multiple deterministic analyses is presented in this paper. The intent is to extract reliability information by conducing only tens instead of millions of deterministic analyses as an alternative to the classical Monte Carlo Simulation. It is particularly applicable when a deterministic analysis requires a considerable amount of computational time to satisfy the underlying physics. It is developed by integrating the second-order reliability method and an improved response surface method by removing its deficiencies. The efficiency of the integrated scheme is further improved by using advanced statistical and factorial schemes producing compounding beneficial effect. The concept is elaborated using two different illustrative examples. To validate the procedure, the underlying reliabilities are estimated by using the basic Monte Carlo simulation technique to develop the reference or benchmark value. Then, the accuracy and efficiency of the method are compared and verified.
\end{abstract}

Keywords Reliability evaluation - Large structural systems · Finite element method · FORM/SORM - Implicit

Achintya Haldar

haldar@u.arizona.edu

Reda Farag

red_bordany@yahoo.com

1 Present Address: Qassim University, Almulyda, Kingdom of Saudi Arabia

2 Housing and Building Research Center, Giza 11511, Arab Republic of Egypt

3 The University of Arizona, Tucson, AZ 85721, USA limit state function $\cdot$ Response surface method $\cdot$ Advanced statistical and factorial schemes

\section{Nomenclature}

$A_{1}, A_{2}$

Cross sectional areas of left and right walls, respectively

AI-RS All-inclusive response surface method

$b_{0}, \quad$ Unknown coefficients of a polynomial to

$b_{i}, b_{i i}$, and $b_{i j}$ be determined

$b \quad$ Span of coupled beam

CCD, SD Central composite design and saturated design, respectively

$d_{b} \quad$ Depth of the coupled beam

$E_{P} \quad$ The Young's or elastic modulus of pile material

E The wall elastic modulus of pile material

$F \quad$ Lateral concentrated force applies at the

$\begin{array}{ll} & \text { pile head } \\ \text { FORM } & \text { First order reliability method }\end{array}$

FEs Finite elements

$\hat{g}(\mathbf{X}) \quad$ Response surface

$g(\mathbf{X}) \quad$ Limit state function

$h \quad$ Story height

$h_{i} \quad$ A chosen factor that defines the

experimental/sample region

$H \quad$ The building total height

I The pile moment of inertia

$I_{1}, I_{2} \quad$ The moment of inertia of left and right walls, respectively

$k \quad$ The number of random variables

$K_{h} \quad$ Soil subgrade reaction

$k_{R} \quad$ The reduced number of random variables

$L \quad$ Distance between the centerlines of left and right walls

LSFs Limit state functions 
$m$

MCS $\quad$ variables

N

$p$

PF

$r$

$\mathrm{RS}$

RSM

SFEM

SORM

$t$

$t_{1}, t_{2}$

$t_{b}$

$u x$

$v$

w

$w_{1}, w_{2}$

$X_{\text {all }}$

$\mathbf{x}_{C_{1}} \mathbf{x}_{C_{2}}$

$\mathbf{x}_{D_{1}}$

$X_{i}(i=$

$1,2, \ldots, k)$

$X_{i}^{C}$

$z$

$\alpha\left(X_{\mathrm{i}}\right)$

$\beta$

$\varepsilon$

$\sigma_{x_{i}}$
Total number of most sensitive random

Monte Carlo Simulation

Number of sample points

The numbers of coefficients necessary to

define a polynomial

The probability of failure

The pile radius

Response surfaces

Response surface method

Stochastic finite element method

Second order reliability method

The pile thickness

The thickness of left and right wall, respectively

Coupled beam thickness

The lateral drift

Poisson's ratio

uniformly distributed Wind loading

Width of left and right walls

The allowable drift

First and second center point

The coordinates of the checking point

The $i$ th random variable, $k$ the number of random variables

The coordinates of the centre point, $i$

The depth

sensitivity indexes of the variable $X_{\mathrm{i}}$

$\beta$-index $=$ Reliability index

Pre-selected convergence criterion

The standard deviation of a random variable $X_{i}$

\section{Introduction}

The state-of-the-art in the reliability or probability of failure $(P F)$ evaluation procedures for Civil/Mechanical structural systems is very advanced. However, $P F$-evaluation of actual structural systems considering their realistic behavior at the time of failure could be challenging, particularly for low probability events. For this class of problems, each deterministic evaluation can be very time consuming. For most of them, the limit state functions (LSFs) also known as performance functions, are expected to be unknown. To study their realistic behavior, the deterministic community generally represents them by finite elements (FEs). Although the required structural behavior can be obtained by the FE analysis using assumed set of values for variables present in the formulation, the LSFs required for the reliability analysis will not be generally available; they are implicit in nature. To extract the

reliability information considering uncertainty in the load and resistance-related variables, representing structural systems using FEs to capture realistic behavior, one can use the basic Monte Carlo simulation (MCS). Suppose, the $P F$ of a system is of the order $10^{-4}$, Haldar and Mahadevan (2000a) suggested that at least $10^{5}$ simulations will be required to extract reasonably accurate reliability information. For the sake of discussion, suppose one deterministic analysis of such system may take around $0.1 \mathrm{~h}$ of computer time, a relatively short time for this discussion. For $10^{5}$ simulations, it will take about $10^{4} \mathrm{~h}$ or 1.14 years of continuous running of a computer. Obviously, it will be impractical to use MCS but clearly indicates the underlying basic challenge. This prompted the authors and their research team members to explore alternative approaches, essentially to find an alternative to the basic MCS.

For reliability estimation with implicit LSFs, several alternatives can be attempted (Haldar and Mahadevan $2000 b)$. They can be grouped into three categories, in the assessment of the research team, based on their essential philosophy. They are (1) MCS, (2) sensitivity-based stochastic finite element method (SFEM), and (3) response surface-based method (RSM). Obviously, several sophisticated methods are available to improve the efficiency in the classical MCS including space-filling variance reduction schemes(Haldar and Mahadevan 2000a), importance sampling (Au and Beck 1999, 2001a), directional sampling (Melchers 1994), subset simulation (Au and Beck 2001b), etc. Success of these schemes is generally considered to be problem dependent, often will require several trial approaches before selecting one, and will require a considerable amount of expertise not expected from an engineer for routine applications. The SFEM, proposed by the team in early eighties (Haldar and Mahadevan 2000b), is also found to be not attractive for this class of problems. The other alternative is RSM (Box et al. 1978) and attracted a lot of attention in the profession recently. The primary purpose of applying RSM in the reliability analysis is to approximate the original complex and implicit limit state function using a simple and explicit polynomial (Bucher and Bourgund 1990; Khuri and Cornell 1996; Yao and Wen 1996). A considerable amount of work is reported in the literature. The works can be grouped into several categories including High Dimensional Model Representation (HDMR) (Rao and Chowdhury 2009; Wei and Rahman 2007), Explicit Design Space DecompositionSupport Vector Machines (EDSD-SVM) (Basudhar and Missoum 2008; Basudhar et al. 2008), artificial neural networks (Hurtado and Alvarez 2000), various variance reduction techniques (Haldar and Mahadevan 2000b), several surrogate approaches including Kriging (Bichon et al. 2008), polynomial chaos (Blatman and Sudret 2010; Dubourg and Sudret 2014), etc. They are summarized in 
(Sudret 2012). Most of these concepts are mathematically very sophisticated and their application potential is application specific. After evaluating most of these approaches, the research team concluded that for routine structural reliability evaluation, they will require additional efforts and expertise not expected from practicing reliability community. The basic challenge is that the explicit expressions for the required limit state functions satisfying required or specified performance requirements required to implement FORM/SORM-based approaches may not be available using these approaches. The same observation was made in (Dubourg and Sudret 2014).

The above discussions clearly point out that in spite of significant development in the reliability evaluation procedures for implicit limit state functions; there are rooms for improvement. Obviously, the classical MCS, with all its elegant schemes and improved computational power, remains an acceptable option. However, its use for the reliability evaluation of time-wise computationally demanding structures is still an open question. The research team proposed several alternative reliability evaluation concepts in this paper, specifically replacing the classical MCS but keeping its basic simplicity. The primary objective of the proposed concept is to extract reliability information for structures requiring extensive computational time by conducting only tens of deterministic analyses satisfying specific performance criteria and retaining the basic simplicity so that it can be used by both deterministic and probabilistic communities.

\section{The Proposed Novel Concept}

To retain the basic simplicity and ease of implementation by the practicing engineering community without considerable expertise in the reliability estimation procedures or knowledge of using parallel computing to implement the basic MCS concept, the authors decided to exploit several desirable features of the first- and second-order reliability methods FORM/SORM in developing the proposed concept. Since RSM-based procedures have significant advantages over MCS and SFEM, the team proposed to integrate FORM/ SORM with RSM so that implicit LSFs can be expressed explicitly, albeit approximately. The proposed concept appears to be very simple, straight forward, and promising. However, its novelty is in the detail and needs to be developed very cautiously, as discussed below.

As will be discussed in Sect. "Selection of sampling points", the experimental sampling points are generally selected for RSM using sigma-point concept without using any information on underlying distribution of random variables even when it is available or in some cases, they are all assumed to be normally distributed. It is known to the profession that distribution information is important for the reliability evaluation. The selection of center points will require information of the failure region. For large structural systems, the selection of center points will be speculative in nature at best and if a response surface (RS) is not generated in the failure region, RSM-based approaches will not be robust enough for routine applications. Also, based on the methods used to generate a RS, it may require a large number of sampling points or deterministic evaluations of the mechanical system. Obviously, considering efficiency, RSM-based approaches suffer the same deficiency as the basic MCS.

In generating the appropriate limit state functions, the information on distribution of random variables present in the formulation must be incorporated. This simple but basic requirement will invalidate the use of basic RSM in the proposed concept. A careful review of the basic RSM concept will reveal that it has three basic but major deficiencies that restrict its use for the Civil/Mechanical structural reliability evaluation. They are: (1) it cannot incorporate distribution information of random variables even when it is available, (2) if the response surface (RS) is not generated in the failure region, it may not be directly applicable or robust, and (3) for realistic structural systems, it may not give the optimal sampling points. The authors proposed to address them in the following ways.

To bring distributional information of random variables and to efficiently locate the failure region for large complicated systems, the authors propose to integrate RSM and SORM. The iterative scheme of SORM will capture the underlying distribution information of random variables and will locate the coordinates of the most probable failure point in the failure region. For real structures, the identification of the failure region is expected to be challenging, however, it can be captured by representing them with finite elements as is commonly practiced by the deterministic community. The integration will eliminate the first two deficiencies. The third deficiency can be removed by using several advanced factorial sampling schemes producing compounding beneficial effect as will be discussed more in detail in Sect. "New Sampling Schemes". To successfully integrate SORM and RSM, the authors propose the following strategies.

\section{Proposed Reliability Analysis Method for Structural Reliability Evaluation}

\section{Step 1-Generate the Required RS by Integrating RSM With SORM}

One of the important topics that deserve initial attention is the characteristics of the response surface (RS) that can be 
used in any SORM-based approach. Three items need to be considered for generating the appropriate RS are: (1) the degree of polynomial to be used to generate it, (2) the location of center points around which sampling points will be selected, and (3) experimental schemes that can be used in selecting sampling points. These items also indicate some of the additional fundamental weaknesses of RSM. They are discussed more formally in the following sections.

To differentiate between the basic RS scheme used in the past and the improved RS scheme proposed by the authors by removing its deficiencies for structural reliability evaluation, the new scheme will be denoted as All Inclusive RS or AI-RS. AI-RS will bring the distribution information of the random variables in the formulation. It will also assure success in generating the required $\mathrm{RS}$ in the failure region.

\section{Selection of Mathematical Form of RSM}

The degree of polynomial used to generate the AI-RS has significant effect on the efficiency of the proposed algorithm, as will be discussed in more detail later. A linear RS may not be appropriate for complicated real structural systems. Higher order polynomial may result in ill-condition of system of equations and exhibit irregular behavior outside of the domain of samples (Gavin and Yau 2008; Sudret 2012). For complicated real structural systems, second-order polynomial, without and with cross terms, are considered to be appropriate. They can be represented as:

$$
\begin{aligned}
& \hat{g}(\mathbf{X})=b_{0}+\sum_{i=1}^{k} b_{i} X_{i}+\sum_{i=1}^{k} b_{i i} X_{i}^{2} \\
& \hat{g}(\mathbf{X})=b_{0}+\sum_{i=1}^{k} b_{i} X_{i}+\sum_{i=1}^{k} b_{i i} X_{i}^{2}+\sum_{i=1}^{k-1} \sum_{j>1}^{k} b_{i j} X_{i} X_{j}
\end{aligned}
$$

where $X_{i}(i=1,2, \ldots, k)$ is the $i$ th random variable, and $b_{0}$, $b_{i}, b_{i i}$, and $b_{i j}$ are unknown coefficients to be determined; they need to be estimated using the response information at the sampling points by conducting several deterministic finite element (FE) analyses and $k$ is the number of random variables in the formulation. The total numbers of coefficient necessary to define Eq. (1) is $p=2 k+1$ and generally it is known as the basic terms. Equation (2) has two components: the basic terms of Eq. 1 and the cross terms. The total numbers of cross terms are $k(k-1) / 2$. The coefficients can be fully defined either by solving a set of linear equations or from regression analysis. The consideration of cross terms is expected to improve the accuracy in the quadratic equation but it can also significantly reduce the efficiency. The authors believe that the intelligent selections of cross terms can increase efficiency without compromising the accuracy. It is an important objective of this paper and will be explored in more detail in the subsequent sections.

\section{Scheme to Locate Center Point}

The response surfaces are generated using sampling points around the center point. Thus, the selection of the center point is very important in the accuracy and efficiency of any reliability evaluation method. Following the SORM scheme, the initial center point will be selected at the mean values $\mu_{i}$ of the random variable $X_{i}$. The response surface $\hat{g}(\mathbf{X})$ can be generated explicitly in terms of the random variables $X_{i}$ 's by conducting deterministic FE analyses at all the experimental sampling points. Once an explicit expression of the limit state function $g(\mathbf{X})$ is obtained, the coordinates of the checking point $\mathbf{x}_{D_{1}}$ (iterative process to identify the coordinates of the most probable failure point) will be available using SORM. The responses can be evaluated again at the checking point $\mathbf{x}_{D_{1}}$, i.e., $\mathrm{g}\left(\mathbf{x}_{D_{1}}\right)$ ), and a new center point $\mathbf{x}_{C_{2}}$ can be selected using a linear interpolation(Bucher and Bourgund 1990; Rajashekhar and Ellingwood 1993) as:

$$
\begin{aligned}
& \mathbf{x}_{C_{2}}=\mathbf{x}_{C_{1}}+\left(\mathbf{x}_{D_{1}}-\mathbf{x}_{C_{1}}\right) g\left(\mathbf{x}_{C_{1}}\right) /\left[g\left(\mathbf{x}_{C_{1}}\right)-g\left(\mathbf{x}_{D_{1}}\right)\right] \\
& \quad \text { if } g\left(\mathbf{x}_{D_{1}}\right) \geq g\left(\mathbf{x}_{C_{1}}\right) \\
& \mathbf{x}_{C_{2}}=\mathbf{x}_{D_{1}}+\left(\mathbf{x}_{C_{1}}-\mathbf{x}_{D_{1}}\right) g\left(\mathbf{x}_{D_{1}}\right)\left[g\left(\mathbf{x}_{D_{1}}\right)-g\left(\mathbf{x}_{C_{1}}\right)\right] \\
& \quad \text { if } g\left(\mathbf{x}_{D_{1}}\right)<g\left(\mathbf{x}_{C_{1}}\right)
\end{aligned}
$$

The new center point $\mathbf{x}_{C_{2}}$ then can be used to develop an explicit performance function for the next iteration. This iterative scheme is repeated until a pre-selected convergence criterion of $\left(\mathbf{x}_{C_{i+1}}-\mathbf{x}_{C_{i}}\right) / \mathbf{x}_{C_{i}} \leq \varepsilon$ is satisfied. The convergence criterion $\varepsilon$ is considered to be $|0.05|$ in this study.

\section{Selection of Sampling Points}

The next step in generating an AI-RS is the selection of sampling points, commonly known as the experimental sampling schemes, around the center point. The concept behind the basic RS sampling scheme can be expressed in the coded variable space as:

$X_{i}=X_{i}^{C} \pm h_{i} \sigma_{x_{i}} \quad i=1,2, \ldots, k$

where $X_{i}^{C}$ and $\sigma_{x_{i}}$ are the coordinates of the center point and the standard deviation of a random variable $X_{i}$, respectively, $h_{i}$ is an arbitrary factor that defines the experimental region, and $k$ is defined earlier. It is to be noted that Eq. 5 does not incorporate any information on the distribution of the random variable. In the context of SORM, AI-RS will be generated in the normal variable 
space. The coordinates of the center point and the standard deviation will be adjusted by considering equivalent normal mean and standard deviation for all non-normal variables present in the formation (Haldar and Mahadevan 2000a).

Available sampling schemes for civil engineering applications can be divided into two categories: saturated design (SD) and a more accurate approach, known as central composite design (CCD) (Box and Wilson 1951; Bucher and Bourgund 1990; Rajashekhar and Ellingwood 1993). The selection of a sampling scheme will dictate the efficiency and accuracy of any AI-RS-based algorithm. Both sampling schemes are shown in Fig. 1 in the coded variable space for $k=3$, (In Fig. $1, k_{R}$ is used instead of $k$ as will be discussed in Sect. "Model reduction by sensitivity analysis").

SD is less accurate but more efficient since it requires only as many sampling points as the total number of unknown coefficients to define the response surface, but it does not have the desired level of accuracy. Using SD, a second-order RS can be generated without and with cross terms and the total number of required sampling points will be $2 k+1$ and $(k+1)(k+2) / 2$, respectively. CCD is more accurate but less efficient. Two major drawbacks of CCD are: (1) it requires cross terms for the second-order polynomial and (2) regression analysis is used to generate the RS requiring too many sampling points. The required number of sampling points for CCD can be shown to be $2^{k}+2 k+1$. The basic three sampling schemes, i.e., SD without and with cross terms and CCD with cross terms, are shown in Fig. 1 for $k=3$. For the ease of discussion, they will be denoted hereafter as Cases 1, 2, and 3, respectively. From the figure, it is very clear that for $k=3$, they will require 7,10 , and 15 sampling points or deterministic evaluations for the three cases, respectively to generate a RS.

Expanding the above discussion for $k$ random variables, one will observe that each of the three cases consists of one center point, $2 k$ axial points, plus $k(k-1) / 2$ edge points for Case 2, and additional $2^{k}$ factorial points for Case 3 . Thus, the total number of sampling points required to generate the necessary AI-RS for the three cases will be $2 k+1,(k+1)(k+2) / 2$ and $2^{k}+2 k+1$, respectively. Since the number of sampling points required for each case is directly related to the efficiency, they are compared in Table 1.

In Table $1, k_{R}$ is used instead of $k$ as will be discussed in Sect. "Model reduction by sensitivity analysis". When the total number of random variables is relatively small, say for $k=5$, the sampling points will be 11,21 , and 43 , respectively, for the three cases. However, if $k$ is increased to 50 representing a large realistic structural system, the corresponding sampling points will be 101, 1326, and $1.1259 \times 10^{15}$, respectively. This exercise clearly indicates that for relatively small problems, the basic SD without and with cross terms can be used; however, the accuracy of the estimated probability of failure for Case 1 cannot be assured. Obviously, for large structural systems, considering efficiency only, CCD will be impractical to implement. However, Cases 2 and 3 need further investigation considering the efficiency and accuracy of the estimated risk.

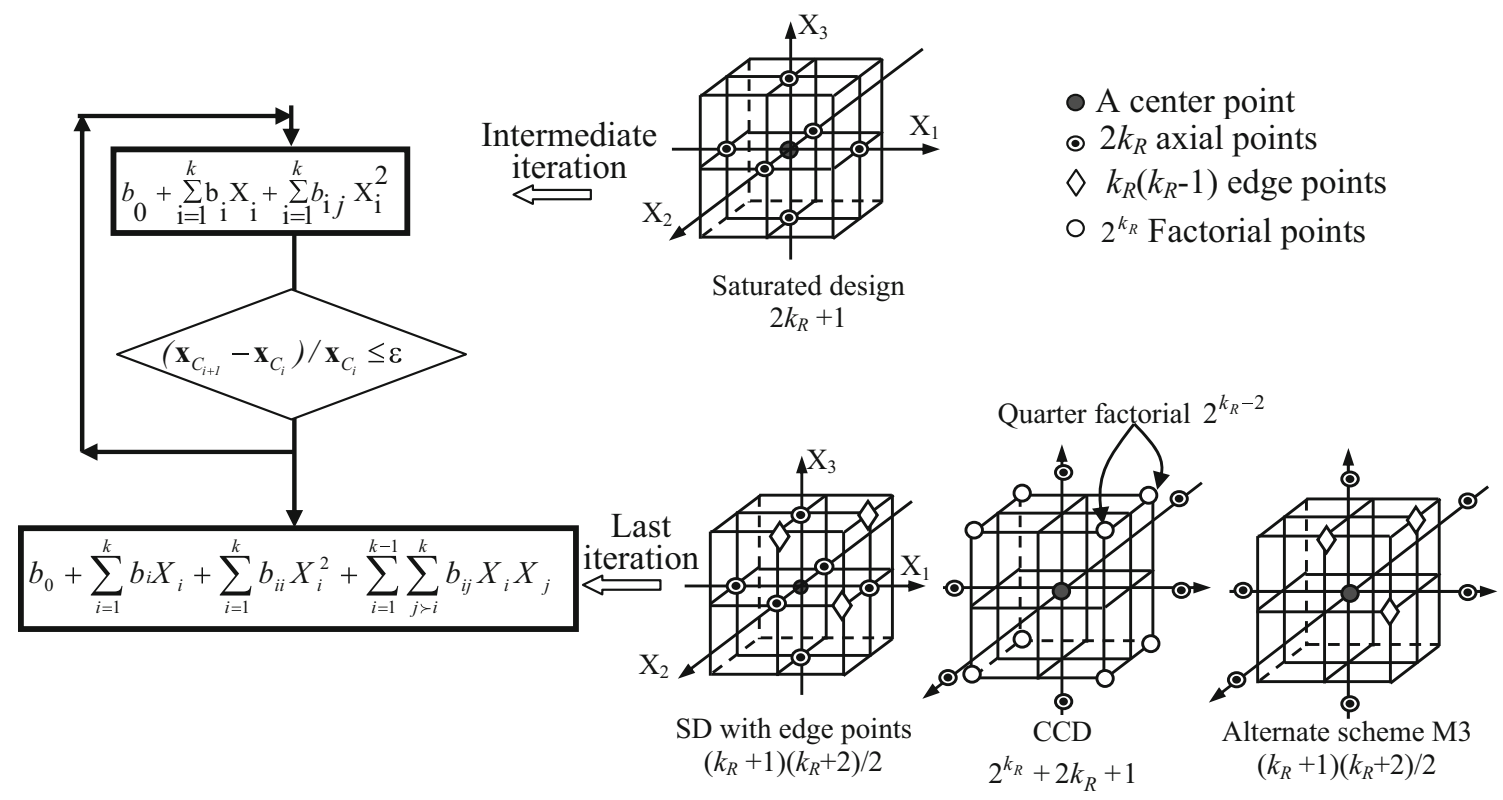

Fig. 1 Algorithm of schemes 2, 3, M2, M3 and alternate scheme M3 (coded variable space $k=3$ ) 
Table 1 Comparison of three cases

\begin{tabular}{|c|c|c|c|c|c|}
\hline Case & $\begin{array}{l}\text { Nos. of random } \\
\text { variables, } k_{R}\end{array}$ & $\begin{array}{l}\text { Nos. of } \\
\text { coefficient, } p\end{array}$ & & $\begin{array}{l}\text { Nos. of sampling } \\
\text { points, } N\end{array}$ & \\
\hline \multirow[t]{2}{*}{1} & 5 & $p=2 k_{R}+1$ & 11 & $N=2 k_{R}+1$ & 11 \\
\hline & 50 & & 101 & & 101 \\
\hline \multirow[t]{2}{*}{2} & 5 & $p=\left(k_{R}+1\right)\left(k_{R}+2\right) / 2$ & 21 & $N=\left(k_{R}+1\right)\left(k_{R}+2\right) / 2$ & 21 \\
\hline & 50 & & 1326 & & 1326 \\
\hline \multirow[t]{2}{*}{3} & 5 & $p=\left(k_{R}+1\right)\left(k_{R}+2\right) / 2$ & 21 & $N=2^{k_{R}}+2 k_{R}+1$ & 43 \\
\hline & 50 & & 1326 & & $1.1259 \times 10^{15}$ \\
\hline \multirow[t]{2}{*}{3 Proposed } & 5 & $p=\left(k_{R}+1\right)\left(k_{R}+2\right) / 2$ & 21 & $N=\left(k_{R}+1\right)\left(k_{R}+2\right) / 2$ & 21 \\
\hline & 50 & & 1326 & & 1326 \\
\hline
\end{tabular}

Bold value indicates too large number of sample points

Conceptually, the required limit state functions can be generated by using the information on the performance criteria for a specific design, obtained either from the design guidelines or specified requirements and the three sampling scheme. Since the LSFs are available in an explicit form at this stage, the reliability information can be extracted using the standard SORM. However, considering accuracy and efficiency, the proposed concept is not yet ready for routine applications since it may still require thousands not tens of deterministic evaluations; it will not satisfy one of the main objectives of the study. This will necessitate exploring Step 2, as discussed below.

\section{Step 2-Development of New Computational and Statistical Concepts to Improve Efficiency of Step 1}

The basic concept discussed in Step 1 is sound and will be applicable for smaller systems with relatively few numbers of random variables. To apply the concept for realistic large structural systems requiring extensive computational time, the authors explored several model reduction, computational and statistical concepts, individually and then combined them to produce the maximum beneficial effect. They are briefly discussed below.

\section{Model Reduction by Sensitivity Analysis}

It is well known that uncertainty in the individual variables affect the stochastic response behavior at the system level quite differently. The uncertainty propagation characteristic of a random variable can be assessed in terms of its sensitivity index (Haldar and Mahadevan 2000a, b). The sensitivity of a variable $X, \alpha(X)$ is the directional cosines of the unit normal variable at the checking or design point. Information on them will be available from SORM analyses. The efficiency can be improved significantly without sacrificing accuracy by considering some of the less significant random variables with smaller sensitivity indexes to be deterministic at their mean values. Based on their experience (Haldar and Mahadevan 2000b), the authors believe that the computational efficiency of the basic concept will be improved significantly requiring less numbers of FE analyses. The parameter $k$ discussed earlier will now be reduced to $k_{R}$. There is no doubt that this reduction will increase efficiency, however, this improvement may not be large enough for complicated large structural systems. In other words, the objective of this step is to reduce the list of candidate random variables to a relatively few so that subsequent experiments will be more efficient and require fewer runs or deterministic evaluations.

\section{New Sampling Schemes}

The proposed concept is iterative in nature. The efficiency of the iterative process can be improved further by capturing advantages of SD and CCD schemes. Less accurate but more efficient schemes can be used for the intermediate iterations followed by the most accurate but inefficient scheme in the final iteration. The authors and their research team examined many such alternatives (Huh and Haldar 2001). Some of the promising schemes are:

Scheme 1 Use SD with 2nd order polynomial without the cross terms (Case 1) throughout all the iterations. This scheme is expected to be the most efficient but least accurate in estimating the probability of failure.

Scheme 2 Use SD with 2nd order polynomial without the cross terms in intermediate iterations (Case 1) and SD with full 2nd order polynomial (Case 2 ) in the final iteration.

Scheme 3 Use SD with 2nd order polynomial without the cross terms in intermediate iterations (Case 1) and CCD with full 2nd order polynomial in the final iteration (Case 3).

The total number of sampling points required to generate the necessary AI-RSs for Schemes 1, 2, and 3 are 
$2 k_{R}+1,\left(k_{R}+1\right)\left(k_{R}+2\right) / 2$ and $2^{k_{R}}+2 k_{R}+1$, respectively, where $k_{R}$ represents the total number of sensitive random variables after making less sensitive variables constants at their mean values. It will be a significant improvement in the efficiency since $k_{R}$ is expected to be much smaller than the total number of random variables $k$ present in the formulation. However, the basic implementation potential of the three schemes still remains the same even with smaller $k_{R}$, i.e., in general they may not be used to estimate reliability of large structural systems, as discussed in more detail next.

For the discussion purpose and to avoid any confusion, the method discussed so far with the three above schemes will be collectively denoted as AI-RSM-SORM.

\section{Step 3 Improve Efficiency of Step 2 Further-Use Advanced Factorial Schemes (AFS)}

Before proposing further improvements, some of the basic features of the three sampling schemes shown in Fig. 1 for three basic cases need further elaboration. It is clear that the total number of basic terms required to generate a second order polynomial, i.e., $2 k_{R}+1$ cannot be changed. However, by the appropriate selection of the edge and factorial points by reducing the simulation region, the efficiency can be significantly improved. The authors consider several options. Some of the most promising approaches are discussed below. Scheme 1 is essentially Case 1. It is the most efficient alternative but may not satisfy the accuracy requirement for large structural systems. It is not consider further for subsequent improvements.

\section{Improvement of Scheme 2-Selection of Edge Points-}

\section{Scheme M2}

As discussed in Sect. "Model Reduction by Sensitivity Analysis", the information on sensitivity indexes was used to reduce the total number of random variables in a formulation from $k$ to $k_{R}$. The authors propose to use the information of sensitivity indexes of $k_{R}$ random variables readily available from SORM to further improve the efficiency of Scheme 2. All $k_{R}$ random variables in the formulation can be arranged in descending order of their sensitivity indexes $\alpha\left(X_{\mathrm{i}}\right)$, i.e., $a\left(X_{1}\right)>a\left(X_{2}\right)>a\left(X_{3}\right)$ $\ldots \ldots>\alpha\left(X_{k_{R}}\right)$. In implementing Scheme 2, SD with second order polynomial without cross terms will be used in all intermediate iterations; however, in the last iteration, the cross terms will be added only for the most sensitive random variable. This is expected to significantly improve efficiency by not considering cross terms for other less significant random variables. However, it will not address the accuracy in the reliability estimation. To address accuracy, other less sensitive random variables can be added one by one in a sequence and the reliability index can be calculated until the changes in the reliability index become negligible. Since it is a modified version of Scheme 2, it will be denoted hereafter as Scheme M2. To discuss the improvement in the efficiency, suppose out of $k_{R}$ random variables, the total number of the most sensitive random variable is $m$. For Scheme M2, the response surface can be expressed as:

$\hat{g}(\mathbf{X})=b_{0}+\sum_{i=1}^{k_{R}} b_{i} X_{i}+\sum_{i=1}^{k_{R}} b_{i i} X_{i}^{2}+\sum_{i=1}^{k_{R}-1} \sum_{j>1}^{m} b_{i j} X_{i} X_{j}$

The total number of sampling points required for Scheme 2 and Scheme M2 will be $\left(k_{R}+1\right)\left(k_{R}+2\right) / 2$ and $2 k_{R}+1+m\left(2 k_{R}-m-1\right) / 2$, respectively. For $k_{R}=5$ and $m=2$, the total number of sampling points will be 21 and 18 , respectively; a modest improvement in the efficiency. However, for $k_{R}=50$ and $m=2$ representing a real structural system, the total number of sampling points will be 1326 and 198, respectively. Obviously, this is a significant improvement in the efficiency. This will be discussed in more detail with the help of examples.

Improvement of Scheme 3-Advanced factorial schemesScheme M3

As in Scheme M2, all $k_{R}$ random variables in the formulation will be arranged in descending order of their sensitivity indexes $\alpha\left(X_{\mathrm{i}}\right)$, i.e. $a\left(X_{1}\right)>a\left(X_{2}\right)>a\left(X_{3}\right)$ $\ldots \ldots>\alpha\left(X_{k_{R}}\right)$..Scheme 3 will be initiated by generating second-order AI-RSM-SORM-Scheme 3 using SD without cross terms for the intermediate iterations, however, in the last iteration, instead of using full factorial plan in CCD (cross terms are required), several fractional factorial schemes (quarter and half) can be used to increase the efficiency (Myers et al. 2009). It is proposed that in the last iteration, only quarter or half of the factorial points instead of full for the most sensitive random variables ( $m$ as in Scheme M2) will be used to develop the response surface in the last iteration. This improved version of Scheme 3 will be denoted hereafter as Scheme M3. It can be shown that if the quarter of the factorial points is used, only $2^{k_{R}-2}+2 k_{R}+1$ number of sample points will be necessary to generate the response surface. For half and full of the factorial points, the total numbers of required sampling points will be $2^{k_{R}-1}+2 k_{R}+1$ and $2^{k_{R}}+2 k_{R}+1$, respectively. For $k_{R}=5$, the required number of sampling points for quarter, half, and full factorial schemes will be 19, 27, and 43 , respectively. For $k_{R}=50$, the corresponding sampling 
points will be $2.81474 \times 10^{14}, \quad 5.6295 \times 10^{14}$, $1.1259 \times 10^{15}$, respectively.

From the above discussion, it is obvious that all the CCD based schemes (full, half and quarter factorial points) will be prohibitive when $k_{R}$ is large. The authors would like to propose another improvement. The concept behind the proposed improvement can be explained with the help of the following example. Since regression equation is used to generate the response surface using CCD, assuming $k_{R}=2$ with two independent variables $X_{1}$ and $X_{2}$, a linear regression equation can be expressed as:

$E(Y \mid \mathbf{X}=\mathbf{x})=E(Y \mid \mathbf{X})=b_{0}+b_{1} X_{1}+b_{2} X_{2}$

where, $b_{0}, b_{1}$, and $b_{2}$ are the regression coefficients needed to define the response surface.

A second-order response surface with two independent variables can be expressed as:

$$
\begin{aligned}
E(Y \mid X)= & b_{0}+b_{1} X_{1}+b_{2} X_{2}+b_{11} X_{1}^{2}+b_{22} X_{2}^{2} \\
& +b_{12} X_{1} X_{2}
\end{aligned}
$$

Equation (8) can be transformed into a linear regression equation by substituting $X_{3}=X_{1}^{2}, X_{4}=X_{2}^{2}, X_{5}=X_{1} X_{2}$, $b_{3}=b_{11}, b_{4}=b_{22}, b_{5}=b_{12}$. Then, Eq. (8) becomes:

$$
\begin{aligned}
E(Y \mid X)= & b_{0}+b_{1} X_{1}+b_{2} X_{2}+b_{3} X_{3}+b_{4} X_{4} \\
& +b_{5} X_{5}
\end{aligned}
$$

In general, any regression model that is linear in the parameters is a linear regression model,

regardless of the shape of the response surface that it represents (Myers et al. 2009). This observation prompted the authors to use edge points instead of factorial points in Scheme 3. The modified scheme will be denoted hereafter as "Alternate Scheme M3". It can be shown that for Alternate Scheme M3, the total numbers of sampling points required will be $\left(k_{R}+1\right)\left(k_{R}+2\right) / 2$. For $k_{R}=5$ and 50 , the required number of sampling will be 21 , and 1326, respectively. Even when $k_{R}=50$, the total number of deterministic evaluations becomes manageable. It is significantly smaller than the numbers of the order of $10^{14}$ required for Scheme M3 discussed earlier.

In order to compare the implementation potential of the aforementioned schemes, the total number of samples required for each scheme is plotted versus the total number of sensitive random variables number, $k_{R}$ in the formulation in Fig. 2. The curve between the points is plotted just to show the trend of improvement in the efficiency. In generating curves for Scheme M2, for a total number of random variables $k_{R}$, the most sensitive random variables $m$ is assumed to be the approximate value of $k_{R} / 2$. When $k_{R}$ is an odd number, $k_{R} / 2$ is rounded up to the next integer. Figure 2 clearly indicates that Scheme 3 and Alternate

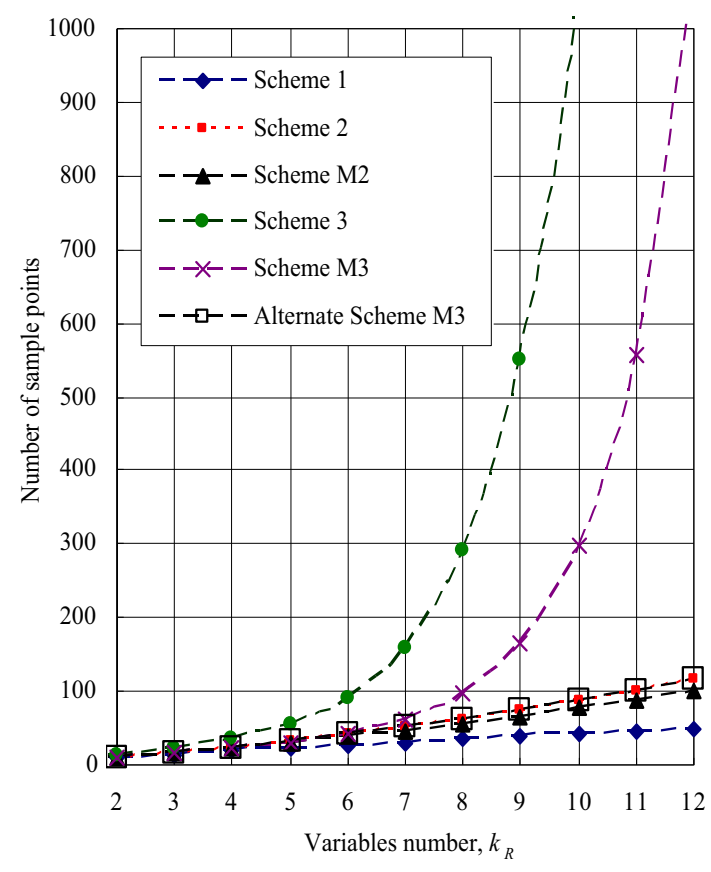

Fig. 2 Number of the required sample points to different schemes

Scheme M3 are now viable options to estimate reliability of large structural systems.

\section{Verification of IRSM-SORM}

Before a new concept can be accepted, it requires verifications. The proposed concept is verified for different types of problem. For this paper, two examples are given; one relatively simple and the other is more difficult and complicated. A commercially available computer program, STATISTICA (StatSoft 2012), is used for the formulation of response surface function. Another commercially available computer program, COMREL (RCP 2003) is used for the reliability evaluation using SORM.

\section{Example 1: Laterally Loaded Pile}

An onshore steel pipe-pile of length $100 \mathrm{~m}$ is considered to be subjected to a lateral force, $F=750 \mathrm{kN}$ to retain a ship in its berth, as shown in Fig. 3. The outside radius, $r$ and the thickness, $t$ of the pile are 30.0 and $1.0 \mathrm{~cm}$, respectively. The elastic modulus of pile made of steel, $E_{P}=2.01 \times 10^{8} \mathrm{kN} / \mathrm{m}^{2}$ and the modulus of soil subgrade reaction, $K_{h}=1500 \mathrm{kN} / \mathrm{m}^{3}$. The statistical characteristics of 5 random variables, as reported in the literature (Bednar 1986; NBoS 1980), are summarized in Table 2.

If the soil-pile system considered here is presented as a beam on elastic foundation, the governing equation can be expressed as (Poulus and Davis 1980): 


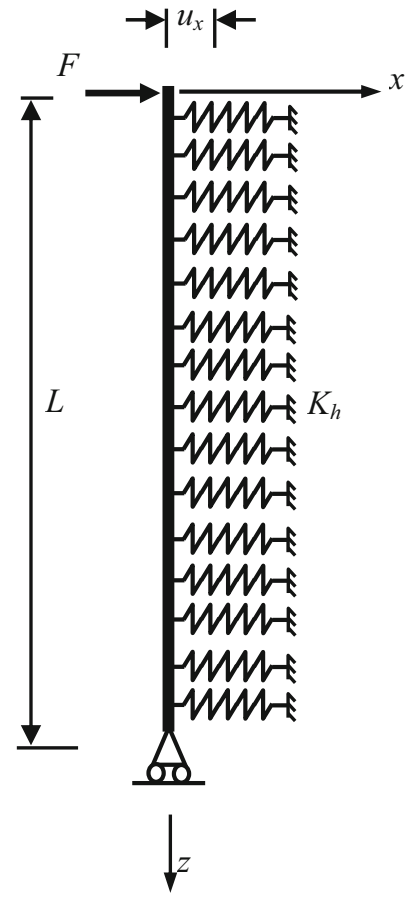

Fig. 3 Laterally loaded pile-example 1

$E_{P} I\left(\mathrm{~d}^{4} u_{x} / \mathrm{d} z^{4}\right)+K_{h} u_{x}=0$

where, $E_{P}$ is elastic modulus of the pile; $I$ is the moment of inertia of the pile section; $u_{x}$ is the lateral drift, $\mathrm{z}$ is the depth, and $K_{h}$ is the lateral subgrade reaction.

Poulus and Davi (Poulus and Davis 1980) suggested an explicit expression to estimate the top drift of the pile. Assuming the permissible drift $X_{\text {all }}$ to be $50 \mathrm{~cm}$, the corresponding limit state function (LSF) for the top drift can be expressed as:

$$
\begin{aligned}
\mathrm{g}(\mathbf{X})= & X_{\text {all }}-u_{x} \\
= & X_{\text {all }}-2 F B /\left(2 K_{h} r\right) \times[\sinh (B L) \cosh (B L) \\
& \quad-\sin (B L) \cos (B L) / D] \\
B= & \left(r K_{h} / 2 E_{P} I\right)^{1 / 4} \text { and } D=\sinh ^{2}(B L)-\sin ^{2}(B L)
\end{aligned}
$$

All the variables were defined earlier.

Representing the pile by two-dimensional (2D) beam elements resting on lateral spring elements and using a commercially available finite element computer program COSMOS/M (Structural Research and Analysis Corporation (SRAC) 2000), the lateral drift at the top of the pile, $u_{x}$ is estimated as $22.58 \mathrm{~cm}$. The same value can be obtained by using Eq. (11). This validates the FE representation.

\section{Reliability Evaluation Using Proposed Different Schemes}

Initially, using SD and second order polynomial without cross terms, an AI-RS was constructed. The sensitivity indexes for $F, K_{h}, r, E$ and $t$ are found to be $-0.950,0.225$, $0.215,0.020$ and 0.016 , respectively. For this illustrative example, $t$ with the lowest sensitivity index is considered to be a deterministic variable at its mean value. The corresponding LSF with 4 random variables for the top lateral drift using Scheme 1 is found to be:

$$
\begin{aligned}
& \hat{g}(\mathbf{X})=50-\left(2.5991 \times 10^{4}+104.40 \times F-77.54\right. \\
& \times K_{h}-9.7881 \times 10^{-4} \times r-6.298 \times 10^{-5} \times E \\
& \left.-1.5844 \times 10^{-2} \times F^{2}+0.175 \times K_{h}^{2}+1.2683 \times 10^{-5} \times r^{2}\right)
\end{aligned}
$$

Similar expressions for LSF for Schemes 2, M2, 3, M3 and Alternate Scheme M3 are generated. The reliability indexes and the corresponding probabilities of failure are then estimated using SORM. The results are summarized in Table 3.

To generate a reference point for comparison, using $10^{5}$ MCS and explicit LSF represented by Eq. (11), the probability of failure is estimated to be $2.32 \times 10^{-2}$, denoted as (MCS-4 RVs) in Table 3. The corresponding reliability index can be shown to be 1.991. If all five random variables are used in generating the LSF, the $P F$ is found to be $2.33 \times 10^{-2}$ (MCS-5 RVs), this is essentially the same as that of with 4 random variables. This exercise clearly indicates the benefit of reducing the size of the problem by using the information on the sensitivity indexes. Using LSF given by Eq. (11) and SORM, the reliability index is found to be 1.990 . For this particular simple problem, the reliability indexes estimated by SORM and MCS with $10^{5}$ simulations using LSF with 4 and 5 random variables are essentially the same. This gives a very reasonable reference

Table 2 Statistical characteristic of random variables- Example 1

\begin{tabular}{lllllllll}
\hline & Random variables & Symbol & Distribution & Nominal & Mean & Bias & COV & Refs. \\
\hline 1 & Lateral load & $F$ & EV-I & $750 \mathrm{kN}$ & 585 & 0.78 & 0.37 & $($ NBS 1980) \\
2 & Radius & $r$ & Ln & $0.30 \mathrm{~m}$ & 0.30 & 1.00 & 0.10 & $($ Bednar 1986) \\
3 & Thickness & $t$ & Ln & $1.0 \mathrm{~cm}$ & 1.0 & 1.00 & 0.05 & $($ Bednar 1986) \\
4 & Pile elastic modulus & $E_{P}$ & Ln & $2.01 \times 10^{8} \mathrm{kN} / \mathrm{m}^{2}$ & $2.01 \times 10^{8}$ & 1.00 & 0.06 & $(\mathrm{NBS} 1980)$ \\
5 & Lateral sub grade reaction & $K_{h}$ & Ln & $1500 \mathrm{kN} / \mathrm{m}^{3}$ & 1725 & $1.15^{\mathrm{a}}$ & $0.21^{\mathrm{a}}$ & \\
\hline
\end{tabular}

a Data not available. Assumed parameters are based on judgment 
Table 3 Results of reliability analysis-example 1

\begin{tabular}{|c|c|c|c|c|c|c|c|c|c|}
\hline & & \multicolumn{5}{|c|}{ Variables sensitivities } & \multirow[t]{2}{*}{$\beta$} & \multirow[t]{2}{*}{$P F$} & \multirow{2}{*}{$\begin{array}{l}\text { No. of calls } \\
N\end{array}$} \\
\hline & & $F$ & $K_{h}$ & $r$ & $E$ & $t$ & & & \\
\hline \multicolumn{10}{|c|}{ (i) Response surface } \\
\hline 1 & Second order polynomial & -0.950 & 0.225 & 0.215 & 0.020 & 0.016 & 2.227 & $1.30 \times 10^{-2}$ & 11 \\
\hline 2 & Scheme 1 & -0.755 & 0.477 & 0.449 & 0.004 & - & 1.248 & $1.06 \times 10^{-2}$ & 9 \\
\hline 3 & Scheme 2 & -0.850 & 0.383 & 0.360 & 0.035 & - & 1.979 & $2.39 \times 10^{-2}$ & 15 \\
\hline \multirow[t]{3}{*}{4} & Scheme M2-1, $H$ & -0.846 & 0.387 & 0.364 & 0.036 & - & 1.972 & $2.43 \times 10^{-2}$ & 12 \\
\hline & Scheme M2-2, $H, K_{h}$ & -0.850 & 0.383 & 0.360 & 0.036 & - & 1.979 & $2.39 \times 10^{-2}$ & 14 \\
\hline & Scheme M2-3, $H, K_{h}, r$ & -0.850 & 0.383 & 0.360 & 0.035 & - & 1.979 & $2.39 \times 10^{-2}$ & 15 \\
\hline \multirow[t]{3}{*}{5} & Scheme 3 & -0.847 & 0.393 & 0.357 & 0.035 & - & 2.003 & $2.26 \times 10^{-2}$ & 25 \\
\hline & Scheme M3, half & -0.857 & 0.398 & 0.325 & -0.027 & - & 2.003 & $2.26 \times 10^{-2}$ & 17 \\
\hline & Quarter & -0.892 & 0.370 & 0.256 & -0.048 & - & 1.998 & $2.29 \times 10^{-2}$ & 13 \\
\hline 6 & Alternate Scheme M3 & -0.892 & 0.370 & 0.256 & -0.048 & - & 2.092 & $1.82 \times 10^{-2}$ & 15 \\
\hline \multicolumn{10}{|c|}{ (ii) Explicit limit state } \\
\hline 7 & Monte Carlo-4 RVs & & & & & & 1.991 & $2.33 \times 10^{-2}$ & $10^{5}$ \\
\hline 8 & SORM-4 RVs & -0.857 & 0.370 & 0.358 & 0.036 & - & 1.991 & $2.32 \times 10^{-2}$ & 1 \\
\hline 9 & Monte Carlo-5 RVs & & & & & & 1.992 & $2.33 \times 10^{-2}$ & $10^{5}$ \\
\hline 10 & SORM-5 RVs & -0.856 & 0.370 & 0.358 & 0.036 & 0.028 & 1.990 & $2.33 \times 10^{-2}$ & 1 \\
\hline
\end{tabular}

value against which other schemes proposed here need to be compared.

The reliability indexes using Schemes 1, 2, and 3 found to be $1.248,1.979$, and 2.003 , respectively, as shown in Table 3. They required 9, 15, and 25 deterministic evaluations, respectively. As expected, Scheme 1 is very efficient but will not satisfy the accuracy requirement. The efficiency of Scheme 2 is improved by considering Scheme M2 by adding the cross terms for the most sensitive variables $F, K_{h}$, and $r$, denoted as Scheme M2-1 (modified Scheme 2 with $F$ as the most significant variable), M2-2 (modified Scheme 2 with $F$ and $K_{h}$ as the most significant variable), and M2-3 (modified Scheme 2 with $F$, $K_{h}$ and $r$ as the most significant variable), respectively, as shown in Table 3. The corresponding reliability indexes are $1.972,1.979$, and 1.979 , requiring 12,14 , and 15 deterministic analyses, respectively. Adding more cross terms to Scheme M2-2 or Scheme M2-3 did not improve the reliability estimation. In all these cases, the estimated reliability indexes are very similar to the reference value of 1.991 and reliability information is extracted using tens of deterministic evaluations.

Reliability indexes using Scheme M3 with half and quarter factorial points and Alternate Scheme M3 are found to be $2.003,1.998$, and 2.092 using 17, 13, and 15 deterministic analyses, as shown in Table 3 . Their accuracy is similar to Scheme 3 using 25 deterministic analyses. Overall, the accuracies in all the schemes in M2 are little inferior to that of all different schemes for M3.

\section{Example 2: Coupled Shear Wall in High Rise Building}

In this example, a more complicated 2-D plane shear walls problem with 11 random variables is considered, as reported in(Smith and Coull 1991). The coupled shear wall in a 20-story building was subjected to a uniformly distributed wind loading of intensity $16.5 \mathrm{kN} / \mathrm{m}$, along the height, as shown in Fig. 4. Information available in the literature is used to characterize the statistical information on all the random variables (JCSS 2000; Mirza and MacGregor 1979), as summarized in Table 4.

The top drift of the coupled shear wall system is suggested as (Smith and Coull 1991):

$$
\begin{aligned}
& u_{x}=\frac{w H^{4}}{8 E I} \times\left\{1-\frac{1}{K^{2}}\left[1-4 /(K \alpha H)^{2}+8 /\left((K \alpha H)^{4}\right.\right.\right. \\
& \cosh (K \alpha H))(1+K \alpha H \sinh (K \alpha H)-\cosh (K \alpha H))]\}
\end{aligned}
$$

where

$$
\begin{aligned}
& \alpha=\left[12 I_{e} L^{2} /\left(b^{3} h I\right)\right]^{0.5}, \quad K=\left[1+A I /\left(A_{1} A_{2} L^{2}\right)\right]^{0.5} \\
& I_{e}=I_{b} /(1+r), \quad r=12 E I_{b} /\left(b^{2} G A\right) \lambda, \quad G=E /(2(1+v))
\end{aligned}
$$

$A=A_{1}+A_{2}, \quad I=I_{1}+I_{2}$

$A_{1}=t_{1} \times w_{1}, \quad A_{2}=t_{2} \times w_{2}$

$I_{1}=t_{1} \times w_{1}^{3} / 12, \quad I_{2}=t_{2} \times w_{2}^{3} / 12$ 
$0.1 \times 0.25 \mathrm{~m}$ rectangular elements

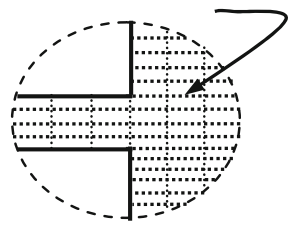

Detail A

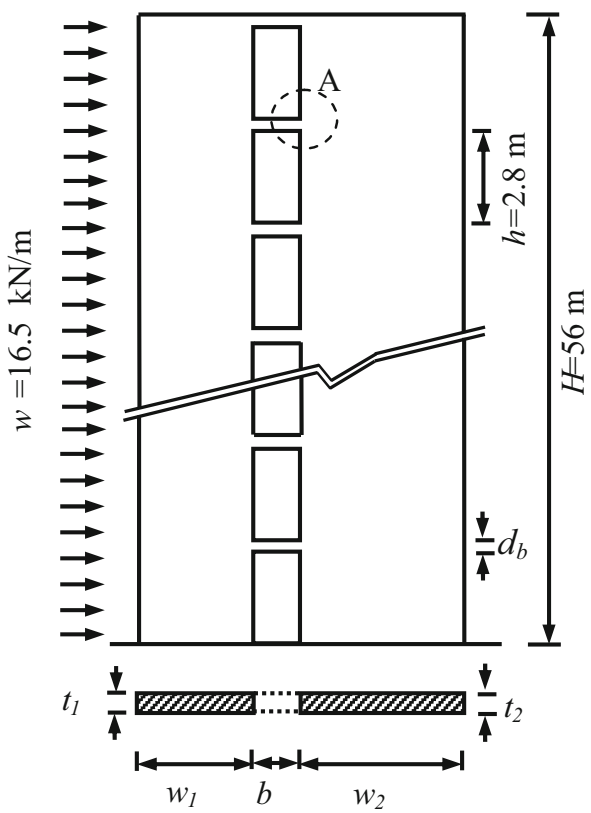

Fig. 4 Coupled shear wall-Example 2

where, $t_{1}, w_{1}, t_{2}$, and $w_{2}$ are the thickness and width of the left and right walls, respectively; $A_{1}, A_{2}, I_{1}$, and $I_{2}$ are the cross sectional areas and moment of inertia of the left and right wall, respectively; $b, L, h$, and $H$ are the clear span of the beams, the distance between the centerlines of the two walls, the story height, and the total height, respectively; $E I_{b}, E I_{e}$ are the true and equivalent flexural rigidity of the connecting beam, respectively; $\lambda=1.2$ represents the cross sectional shape factor for shear for the coupled beam of a rectangular cross section; and $E, G, v$ are the modulus of elasticity, the shear modulus and Poisson's ratio, respectively.

\section{Problem Simplification}

The drift $u_{x}$ is found to be $1.57 \mathrm{~cm}$ when the coupled shear wall was discretized by $0.1 \times 0.25 \mathrm{~m}$ rectangular elements using COSMOS (Structural Research and Analysis Corporation (SRAC) 2000), as shown in Fig. 4. The drift is estimated to be $1.6 \mathrm{~cm}$ using Eq. (14). The allowable drift is assumed to be H/2000 expressed in $\mathrm{cm}$.

For this example with 11 random variables, to generate second-order AI-RSs with SD without and with cross terms will require the estimation of 23 and 78 coefficients, respectively, a relatively challenging task. To minimize the computational effort, initially only a first-order AI-RS is generated requiring the estimation of 12 coefficients, and the sensitivity indexes of all 11 random variables are calculated. Only four variables $w, E, w_{2}$, and $t_{2}$ with sensitivity indexes of $-0.983,0.180$. 0.023, and 0.23, respectively, are considered to be significant random variables and all remaining other seven variables are considered to be deterministic at their mean values. The reliability index using this first-order AI-RS is estimated to be 2.378 .

Using Scheme 1, i.e., SD with second-order polynomial without cross terms, the LSF for the lateral deflection at the top of the frame is expressed as:

Table 4 Statistical characteristic of random variables-example 2

\begin{tabular}{|c|c|c|c|c|c|c|c|c|}
\hline & Random variables & Sym. & Dist. & Nominal & Mean & Bias & $\mathrm{COV}$ & Refs. \\
\hline 1 & Wind loading & $w$ & EV-I & $16.5 \mathrm{kN} / \mathrm{m}$ & 12.87 & 0.78 & 0.37 & (NBS 1980) \\
\hline 2 & The elastic modulus & $E$ & $\mathrm{Ln}$ & $36000 \mathrm{MPa}$ & 36000 & 1.0 & 0.15 & (JCSS 2000) \\
\hline 3 & Poisson's ratio & $v$ & $\mathrm{Ln}$ & 0.15 & 0.15 & 1.0 & 0.18 & a \\
\hline 4 & Left wall width & $w_{1}$ & $\mathrm{~N}$ & $5.00 \mathrm{~m}$ & 5.00 & 1.0 & 0.05 & $\mathrm{a}$ \\
\hline 5 & Left wall thickness & $t_{1}$ & $\mathrm{~N}$ & $0.30 \mathrm{~m}$ & 0.30 & 1.0 & 0.10 & $\mathrm{a}$ \\
\hline 6 & Right wall width & $w_{2}$ & $\mathrm{~N}$ & $7.00 \mathrm{~m}$ & 7.00 & 1.0 & 0.05 & $\mathrm{a}$ \\
\hline 7 & Right wall thickness & $t_{2}$ & $\mathrm{~N}$ & $0.30 \mathrm{~m}$ & 0.30 & 1.0 & 0.10 & $\mathrm{a}$ \\
\hline 8 & Coupled beam depth & $d_{b}$ & $\mathrm{~N}$ & $0.40 \mathrm{~m}$ & 0.40 & 1.0 & 0.02 & (Mirza and MacGregor 1979) \\
\hline 9 & Coupled beam thickness & $t_{b}$ & $\mathrm{~N}$ & $0.30 \mathrm{~m}$ & 0.30 & 1.0 & 0.02 & (Mirza and MacGregor 1979) \\
\hline 10 & Span of coupled beam & $b$ & $\mathrm{~N}$ & $2.00 \mathrm{~m}$ & 2.00 & 1.0 & 0.01 & (Mirza and MacGregor 1979) \\
\hline 11 & Centerline distance & $L$ & $\mathrm{~N}$ & $8.00 \mathrm{~m}$ & 8.00 & 1.0 & 0.01 & (Mirza and MacGregor 1979) \\
\hline 12 & Story height & $h$ & Deterministic & $2.80 \mathrm{~m}$ & & & & \\
\hline
\end{tabular}

a Data not available. Assumed parameters are based on engineering judgment 
Table 5 Results of reliability analysis-example 2

\begin{tabular}{|c|c|c|c|c|c|c|c|c|}
\hline & & \multicolumn{4}{|c|}{ Variables sensitivities } & \multirow[t]{2}{*}{$\beta$} & \multirow[t]{2}{*}{$P F$} & \multirow[t]{2}{*}{$N$} \\
\hline & & $w$ & $E$ & $w_{2}$ & $t_{2}$ & & & \\
\hline \multicolumn{9}{|c|}{ (i) Response surface } \\
\hline 1 & First order polynomial & -0.983 & 0.180 & 0.023 & 0.023 & 2.378 & $8.71 \times 10^{-3}$ & 23 \\
\hline 2 & Scheme 1 & -0.927 & 0.370 & 0.045 & 0.047 & 2.205 & $1.37 \times 10^{-2}$ & 9 \\
\hline \multirow[t]{4}{*}{3} & Scheme 2 & -0.920 & -0.380 & 0.063 & 0.069 & 2.193 & $1.42 \times 10^{-2}$ & 15 \\
\hline & Scheme M2-1 $w$ & -0.925 & -0.374 & 0.050 & 0.054 & 2.198 & $1.40 \times 10^{-2}$ & 12 \\
\hline & Scheme M2-2 $\quad w, E$, & -0.921 & -0.379 & 0.062 & 0.068 & 2.193 & $1.41 \times 10^{-2}$ & 14 \\
\hline & Scheme M2-3 $\quad w, E, w_{2}$ & -0.920 & -0.380 & 0.063 & 0.069 & 2.193 & $1.42 \times 10^{-2}$ & 15 \\
\hline 5 & Scheme 3, & -0.937 & -0.344 & 0.043 & 0.047 & 2.119 & $1.70 \times 10^{-2}$ & 25 \\
\hline \multirow[t]{3}{*}{6} & Scheme M3 & & & & & & & \\
\hline & Half & -0.929 & -0.365 & 0.410 & 0.044 & 2.101 & $1.78 \times 10^{-2}$ & 17 \\
\hline & Quarter & -0.103 & 0.161 & -0.280 & 0.941 & 0.946 & $1.72 \times 10^{-1}$ & 13 \\
\hline 7 & Alternate Scheme M3 & -0.941 & 0.337 & 0.014 & 0.011 & 2.104 & $1.77 \times 10^{-2}$ & 15 \\
\hline \multicolumn{9}{|c|}{ (ii) Explicit limit state } \\
\hline 8 & Monte Carlo-4 RVs & & & & & 2.209 & $1.36 \times 10^{-2}$ & $10^{5}$ \\
\hline 9 & SORM-4 RVs & -0.921 & 0.382 & 0.046 & 0.047 & 2.210 & $1.35 \times 10^{-2}$ & 1 \\
\hline 10 & Monte Carlo-11 RVs & & & & & 2.205 & $1.37 \times 10^{-2}$ & $10^{5}$ \\
\hline 11 & SORM-11 RVs & -0.920 & 0.381 & 0.046 & 0.049 & 2.206 & $1.37 \times 10^{-2}$ & 1 \\
\hline
\end{tabular}

$$
\begin{aligned}
\hat{g}(\mathbf{X})= & H / 2000-\left(-1875.64+22.26 \times w-8.59 \times 10^{-6}\right. \\
\times E & +518.66 \times w_{2}+2.5081 \times 10^{-3} \times t_{2} \\
& \left.-0.22 \times w^{2}-39.66 \times w_{2}^{2}-4.7780 \times 10^{3} \times t_{2}^{2}\right)
\end{aligned}
$$

Similar to the previous example, the reliability index is estimated to be 2.210 using Eq. (20) and SORM. The corresponding reliability index using $10^{5} \mathrm{MCS}$ is estimated to be 2.209. If all 11 random variables are considered in the LSF, the corresponding reliability indexes using SORM and MCS are estimated to be 2.206 and 2.205, respectively. Obviously, using only 4 instead of 11 did not change the estimation of the reliability indexes considerably. The information is summarized in Table 5. For this particular example, the reliability index is found to be 2.205 using only 9 deterministic evaluations using Scheme 1 (SD without cross terms in all iterations) and SORM indicating Scheme 1 is good enough. This indicates that Scheme 1 need not be completely eliminated for further consideration. As in Example 1, the reliability indexes are also estimated using Schemes 1, 2, 3, M2 with three different schemes, M3 with half and quarter factorial points and Alternate Scheme M3. The results are summarized in Table 5 .

Several important observations can be made from the results. Scheme M3 (quarter) failed to estimate the reliability index. The authors made similar observations dealing with other problems. They will not recommend it if it is used alone for the reliability estimation at this stage of their study. For this illustrative complicated structural problem, to reduce the size of the problem, the use of sensitivity indexes using the first-order AI-RS is reasonable. Except for Scheme M3 with quarter factorials points, reliability indexes obtained for all different schemes are found to be very similar to the reference value considered to be 2.209 obtained by $10^{5}$ MCS with 4 random variables. The deterministic evaluations for all these alternatives varied between 9 and 25. The results clearly indicate that the information on the reliability index can be extracted with the reasonable accuracy for relatively small and large structural problems using only tens of deterministic evaluations at highly intelligently selected points using the proposed novel concept. The authors believe that they proposed a novel alternative to classical MCS.

\section{Conclusions}

A novel concept is proposed to estimate the reliability of real large Civil/Mechanical systems represented by finite elements. The intent of the proposed concept is to extract reliability information by conducing only tens of deterministic evaluations at very intelligently selected points instead of thousands or millions particularly for structural systems represented by finite elements to satisfy realistic behavior requiring extensive computer time for a deterministic analysis. It integrates the basic SORM and a modified RSM concept by eliminating its three basic weaknesses but keeps the basic simplicity for everyday use by practicing engineers. It is denoted as AI-RSM-SORM in this paper. The efficiency of the integrated scheme is improved further by using 
sensitivity analysis and several advanced computational, statistical, and factorial schemes. A new concept, denoted as Alternate Scheme M3 is proposed to generate a response surface using CCD by using edge points instead of factorial points. Accuracy and efficiency of all the schemes are verified with the help of two examples; one is relatively simple and the other is more complicated. For both examples, the underlying reliabilities are estimated by using the basic MCS since the necessary limit state functions can be expressed mathematically in close form. The information is used to develop the reference or benchmark value. The systems are then represented by finite elements and all the schemes are used to estimate the underlying reliability. The accuracies of all the schemes are established by comparing the results with the reference value. Schemes M2 and Alternate Scheme M3 appeared to be very accurate and efficient. Both schemes extract reliability information using only tens of deterministic evaluations at very intelligently selected points without sacrificing accuracy, for both relatively small and relatively large structural systems considered in the paper. The concept provides an alternative to classical MCS, the major objective of the paper.

Acknowledgements This study is based on work partly supported by the General Administration of Scientific Mission, the Representative of Higher Education Authority in Egypt. The work is also partly supported by the National Science Foundation under Grant No. CMMI-1403844. Any opinions, findings, conclusions, or recommendations expressed in this paper are those of the writers and do not necessarily reflect the views of the sponsors.

\section{References}

Au K, Beck L (1999) A new adaptive importance sampling scheme for reliability calculations. Struct Saf 21:135-158. doi:10.1016/S0167-4730(99)00014-4

Au K, Beck L (2001a) First excursion probabilities for linear systems by very efficient importance sampling. Probab Eng Mech 16:193-207. doi:10.1016/S0266-8920(01)00002-9

Au SK, Beck JL (2001b) Estimation of small failure probabilities in high dimensions by subset simulation. Probab Eng Mech 16:263-277. doi:10.1016/S0266-8920(01)00019-4

Basudhar A, Missoum S (2008) Adaptive explicit decision functions for probabilistic design and optimization using support vector machines. Comput Struct 86:1904-1917. doi:10.1016/j.comp struc.2008.02.008

Basudhar A, Missoum S, Sanchez AH (2008) Limit state function identification using support vector machines for discontinuous responses and disjoint failure domains. Probab Eng Mech 23:1-11. doi:10.1016/j.probengmech.2007.08.004

Bednar HH (1986) Pressure vessel design handbook. Van Nostrand Reinhold, Malabar, Florida

Bichon B, Eldred M, Swiler L, Mahadevan S, McFarland J (2008) Efficient global reliability analysis for nonlinear implicit performance functions. AAIA J 46:2459-2468

Blatman G, Sudret B (2010) An adaptive algorithm to build up sparse polynomial chaos expansions for stochastic finite element analysis. Probab Eng Mech 25:183-197
Box GEP, Wilson KB (1951) On the experimental attainment of optimal conditions. J R Stat Soc Ser B 13:1-45

Box GE, Hunter WG, Hunter JS (1978) Statistics for experimenters: an introduction to design, data analysis, and model building. Wiley, New York

Bucher CG, Bourgund U (1990) A fast and efficient response surface approach for structural reliability problems. Struct Saf 7:57-66. doi:10.1016/0167-4730(90)90012-E

Dubourg V, Sudret B (2014) Meta-model-based importance sampling for reliability sensitivity analysis. Struct Saf 49:27-36

Gavin HP, Yau SC (2008) High-order limit state functions in the response surface method for structural reliability analysis. Struct Saf 30:162-179. doi:10.1016/j.strusafe.2006.10.003

Haldar A, Mahadevan S (2000a) Probability, reliability and statistical methods in engineering design, 1st edn. Wiley, New York

Haldar A, Mahadevan S (2000b) Reliability assessment using stochastic finite element analysis. Wiley, New York

Huh J, Haldar A (2001) Stochastic finite-element-based seismic risk of nonlinear structures. J Struct Eng 127:323-329. doi:10.1061/ (ASCE)0733-9445(2001)127:3(323)

Hurtado J, Alvarez D (2000) Reliability assessment of structural systems using neural networks. In: Proceedings of the European congress on computational methods in applied sciences and engineering, ECCOMAS 2000, Barcelona

JCSS (2000) Probabilistic Model Code, Part 3: material properties. www.jcss.ethz.ch. Accessed 15 Mar 2013

Khuri AI, Cornell JA (1996) Response surfaces: designs and analyses, Second edn. Marcel Dekker, New York

Melchers RE (1994) Structural system reliability assessment using directional simulation. Struct Saf 16:23-37. doi:10.1016/01674730(94)00026-M

Mirza S, MacGregor J (1979) Variations in dimensions of reinforced concrete members. J Struct Eng 105:751-766

Myers R, Montgomery D, Cook CA (2009) Response surface methodology: process and product optimization using designed experiments. Wiley, New York

NBS (1980) Development of a Probability Based Load Criterion for American National Standard A58: Building Code Requirements for Minimum Design Loads in Buildings and Other Structures. US Department of Commerce

Poulus H, Davis E (1980) Pile Foundation Analysis and Design. John Wiley and Sons, New York

Rajashekhar MR, Ellingwood BR (1993) A new look at the response surface approach for reliability analysis. Struct Saf 12:205-220. doi:10.1016/0167-4730(93)90003-J

Rao BN, Chowdhury R (2009) Enhanced high-dimensional model representation for reliability analysis. Int $\mathrm{J}$ Numer Meth Eng 77:719-750. doi:10.1002/nme.2440

RCP (2003) Reliability Consulting Programs vol V. 6.10, 6.10 edn, Munich, Germany

Smith B, Coull A (1991) Tall building structures: analysis and design. Wily, New York

StatSoft (2012) STATISTICA. Statsoft Inc., http://www.statsoft.com. Accessed 15 Apr 2013

Structural Research and Analysis Corporation (SRAC) (2000) COSMOSM vol V. 2.6. Santa Monica, California

Sudret B (2012) Meta-models for structural reliability and uncertainty quantification. In: Phoon K BM, Quek S, Pang S, editors (ed) Proceedings of the 5th Asian-Pacific symposium on structure reliability (APSSRA'2012). Singapore, pp 53-76

Wei D, Rahman S (2007) Structural reliability analysis by univariate decomposition and numerical integration. Probab Eng Mech 22:27-38. doi:10.1016/j.probengmech.2006.05.004

Yao T, Wen Y (1996) Response surface method for time-variant reliability analysis. J Struct Eng 122:193-201. doi:10.1061/ (ASCE)0733-9445(1996)122:2(193) 